\title{
PAPR reduction of OFDM signals using PTS: a real-valued genetic approach
}

\author{
Jenn-Kaie Lain*, Shi-Yi Wu and Po-Hui Yang
}

\begin{abstract}
The partial transmit sequences (PTS) scheme achieves an excellent peak-to-average power ratio (PAPR) reduction performance of orthogonal frequency division multiplexing (OFDM) signals at the cost of exhaustively searching all possible rotation phase combinations, resulting in high computational complexity. Several researchers have proposed using binary-coded genetic algorithms (BGA) PTS to reduce both the PAPR and computational load. To improve the PAPR statistics of OFDM signals further while still reducing the computational complexity, this paper proposes a new PTS using the real-valued genetic algorithm (RVGA). By defining a cost function based on the amount of PAPR, PTS can be formulated as an optimization problem over a multidimensional real space and solved by implementing the RVGA method. The simulation results show that the performance of the proposed RVGA PTS, along with an extinction and immigration strategy, provides approximately the same PAPR statistic as the exhaustive PTS scheme, while maintaining a low computational load.
\end{abstract}

Keywords: genetic algorithm, orthogonal frequency division multiplexing, partial transmit sequences

\section{Introduction}

Orthogonal frequency division multiplexing (OFDM) is an attractive technique for achieving high-bit-rate wireless communication [1] and has been applied extensively to digital transmission, such as in wireless local area networks and digital video and audio broadcasting systems. Moreover, OFDM has been regarded as a promising transmission technique for next generation wireless mobile communication. However, due to its multicarrier nature, one of the major drawbacks in OFDM systems is the high PAPR, causing high out-of-band radiation when OFDM signals are passed through a radio frequency power amplifier. A number of approaches have been proposed to solve the PAPR problem in OFDM [2]. Among these methods, the PTS is one of the most attractive schemes because of high-quality PAPR reduction performance with no restrictions to the number of subcarriers [3]. In the PTS scheme, the input symbols are partitioned into several disjoint subblocks. Inverse fast Fourier transform (IFFT) is applied to each disjoint subblock, and each corresponding time-domain signal is

\footnotetext{
* Correspondence: lainjk@yuntech.edu.tw
Department of Electronic Engineering, National Yunlin University of Science
and Technology, 123 University Road, Section 3, Douliou, Yunlin 64002, * Correspondence: lainjk@yuntech.edu.tw
Department of Electronic Engineering, National Yunlin University of Science
and Technology, 123 University Road, Section 3, Douliou, Yunlin 64002,

* Correspondence: lainjk@yuntech.edu.tw
Department of Electronic Engineering, National Yunlin University of Science
and Technology, 123 University Road, Section 3, Douliou, Yunlin 64002, Taiwan
}

multiplied by a rotation phase. The objective of the PTS scheme is to select the rotation phases such that the PAPR of the combined time-domain signal is minimized. Increasing exponentially with the number of subblocks and the number of the rotation phases that can be chosen, the searching complexity to find the optimal phases becomes intractable and impractical.

To reduce the computational complexity for searching rotation phases in PTS, various suboptimal methods that achieve significant reduction in complexity were presented in [4-11]. Owing to an intensive improvement of circuit design for genetic algorithms (GAs) in recent years $[12,13]$, PTS based on GAs not only has moderate PAPR reduction performance but also shows potential for practical implementation among these methods. The GA has proved to be a robust, domain-independent mechanism for numeric and symbolic optimization. With the trend of GA hardware becoming more popular and low-priced, the PTS based on GA may provide a practical and economical approach toward solving the difficulty of high PAPR in OFDM systems. Previous studies have demonstrated that the BGA PTS achieves a moderate PAPR reduction in discrete domains [7-9]. However, rotation phases involved in this phase-searching problem are real-valued radians. This prompts 
consideration of a novel implementation of PTS to reduce the PAPR based on a real-valued genetic algorithm (RVGA) method. In the proposed RVGA method, a cost function related to the amount of PAPR is first defined. The cost function is then translated into a realvalued parameter optimization problem, which can be solved effectively by the RVGA. The simulation results show that the performance of the proposed RVGA PTS along with an extinction and immigration strategy provides a PAPR statistic approaching that of the exhaustive PTS while maintaining a low computational load.

The rest of this paper is organized as follows. Section 2 presents a description of the OFDM system and formulates the PTS PAPR reduction problem as a combinatorial optimization problem over a multidimensional real space. Section 3 describes how to solve this problem using the RVGA method along with an extinction and immigration strategy. Section 4 describes the simulative results and discussion. Finally, conclusions are drawn in Section 5.

\section{System model and problem formulation}

\subsection{OFDM systems and PAPR definition}

In an OFDM system with $N$ subcarriers, the discretetime transmitted signal is given by

$$
x_{k}=\frac{1}{\sqrt{N}} \sum_{n=0}^{N-1} X_{n} e^{j \frac{2 \pi n k}{f_{s} N}}, \quad k=1,2, \ldots, \quad f_{s} N-1
$$

where $j=\sqrt{-1}, X_{n}$ are input symbols modulated by PSK or QAM, and $f_{s}$ is an over-sampling factor to simulate the behavior of continuous signals. The PAPR of the transmitted signal in (1), defined as the ratio of the maximum to the average power, can be expressed by

$$
\text { PAPR }=10 \log _{10} \frac{\max \left|x_{k}\right|^{2}}{E\left[\left|x_{k}\right|^{2}\right]}(\mathrm{dB}),
$$

where $E[$.$] denotes expectation operation.$

\subsection{Formulation of OFDM with PTS}

The functional block diagram of an OFDM system with a PTS scheme is shown in Figure 1 as that in [4]. The data block $\mathbf{X}$ is partitioned into $M$ disjoint subblocks $\mathbf{X}_{m}$, where $m=1,2, \ldots, M$, such that

$$
\mathbf{X}=\sum_{m=1}^{M} \mathbf{X}_{m}
$$

Here, it is assumed that the subblocks $\mathbf{X}_{m}$ consist of a set of subcarriers of equal size $N$. The partitioned subblocks are converted from the frequency domain to the time domain using $N$-point IFFT. Due to IFFT being a linear transformation, the representation of the block in the time domain is given by

$$
\mathbf{x}=\operatorname{IFFT}\left\{\sum_{m=1}^{M} X_{m}\right\}=\sum_{m=1}^{M} \operatorname{IFFT}\left\{\mathbf{X}_{m}\right\}=\sum_{m=1}^{M} \mathbf{x}_{m} .
$$

The goal of the PTS is to form a weighted combination of the $M$ time-domain partial sequences $\mathbf{x}_{m}$ by a rotation vector $\mathbf{b}=\left[\begin{array}{llll}b_{1} b_{2} & \ldots & b_{M}\end{array}\right]$ to minimize the PAPR, which is given by

$$
\mathbf{x}^{\prime}(\mathbf{b})=\sum_{m=1}^{M} b_{m} \mathbf{x}_{m}
$$

To minimize the peak power of $\mathbf{x}^{\prime}$, each partial sequences $\mathbf{x}_{m}$ should be properly rotated. Letting $b_{m}=$ $e^{j \varphi m}$, where $\varphi_{m}$ can be chosen freely within $[0,2 \pi),(5)$ can be expressed as

$$
\mathbf{x}^{\prime}(\boldsymbol{\Phi})=\sum_{m=1}^{M} e^{j \phi_{m}} \mathbf{x}_{m}
$$

where $\Phi=\left[\begin{array}{lll}\varphi_{1} \varphi_{2} & \ldots & \varphi_{M}\end{array}\right]$. Here, the objective of the PTS scheme is to design a rotation phase vector $\boldsymbol{\Phi}$ that minimizes the PAPR. PAPR reduction with the PTS technique is related to the problem of minimizing $\max \mid$ $\mathbf{x}^{\prime}(\Phi) \mid$ subject to $0 \leq \varphi_{m} \leq 2 \pi, m=1,2, \ldots, M$, and however, it is equivalent to an exhaustive search for a combinatorial optimization problem, which requires an enormous amount of computations to search all over possible candidate rotation phase vectors.

\section{The real-valued genetic algorithm PTS \\ 3.1 RVGA PTS}

By translating the phase-searching problem of the PTS into a real-valued parameter optimization, this study proposes using the RVGA to find a rotation phase vector to reduce PAPR. This study associates every rotation phase vector using a chromosome to apply the RVGA to the PTS PAPR reduction problem. The following delineates the steps involved in the RVGA PTS.

Step 0-Initialization: To begin the RVGA PTS, this study defines an initial population of $P$ chromosomes, where $P$ is the population size. Each chromosome contains $M$ genes, in which the gene values $\varphi_{i}$ are rotation phases initially selected at random. The range of gene values is between $\varphi_{l o}$ ans $\varphi_{h i}$, then the gene values are initialized by

$$
\phi_{i}=\left(\phi_{h i}-\phi_{l o}\right) u+\phi_{l o}
$$

where $\varphi_{h i}, \varphi_{l o}$, and $u$ are the highest value in the variable range, the lowest value in the variable range, and a uniformly distributed random variable in $[0,1]$. In the PTS scheme, the values of $\varphi_{l o}$ and $\varphi_{h i}$ are set at 0 and 


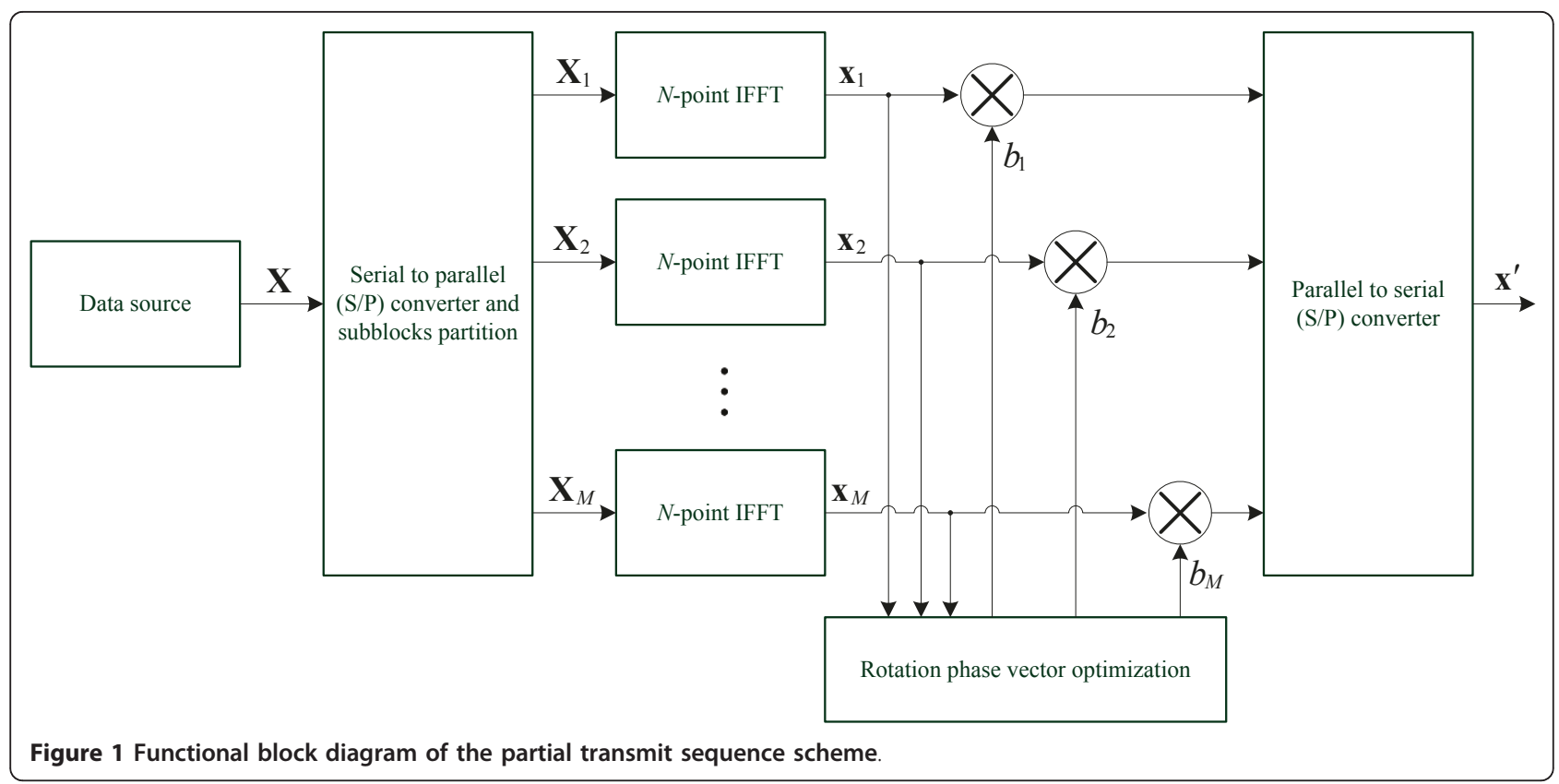

$2 \pi$, respectively. Given an initial population of $P$ chromosomes, the full matrix of $P \times M$ random rotation phases is generated.

Step 1-Evaluation and Selection: In each generation, the cost values are computed for each of the $P$ chromosomes by substituting the corresponding rotation phase vector $\boldsymbol{\Phi}$ into the cost function of $\max \left|\mathbf{x}^{\prime}(\boldsymbol{\Phi})\right|$. Thereafter, the $T$ chromosomes with the lowest cost values are chosen for a mating pool, from which two chromosomes are selected according to a roulette wheel selection for the next crossover step [14].

Step 2-Crossover: Crossover is a recombination operation that combines subparts of two parent chromosomes to exchange the genetic material between chromosomes. A crossover probability $p_{c}$ controls the degree of crossover. A $1 \times M$ sequence, often referred to as a crossover mask, is constructed, consisting of $1 \mathrm{~s}$ generated with crossover probability $p_{c}$ and 0 s generated with probability $\left(1-p_{c}\right)$. When the elements in the crossover mask are $1 \mathrm{~s}$, the genes of the two parent chromosomes in the corresponding positions will be mixed with each other, where if they are 0s, the corresponding genes will be unchanged. Suppose $\boldsymbol{\Phi}_{1}$ and $\boldsymbol{\Phi}_{2}$ are two parents selected, the $i$ th element in the crossover mask is 1 , and $\varphi_{1, i}$ and $\varphi_{2, i}$ are the $i$ th genes in $\boldsymbol{\Phi}_{1}$ and $\boldsymbol{\Phi}_{2}$, respectively. The $i$ th genes in the next generation of $\boldsymbol{\Phi}_{1}$ and $\boldsymbol{\Phi}_{2}$ are $r \varphi_{1, i}+(1-r) \varphi_{2, i}$ and $r \varphi_{2, i}+$ $(1-r) \varphi_{1, i}$, respectively, where $r$ is a uniformly distributed random variable in $[0,1]$. This crossover operation will repeat until the number of the new population size reaches $P$.
Step 3-Mutation: To explore more regions within the solution space, mutation should be adopted in the RVGA method [14]. This study constructs a $1 \times P$ mutation mask sequence, consisting of 1 s generated with the mutation probability $p_{m}$ and 0 s generated with probability $\left(1-p_{m}\right)$, for all chromosomes in each generation. When the elements in the mutation mask are $1 \mathrm{~s}$, the genes of the chromosome in the corresponding positions will change. However, if they are 0s, the corresponding genes will remain unchanged. Supposing the $i$ th element $\varphi_{i}$ in rotation phase vector $\boldsymbol{\Phi}$ is selected for mutation, (7) can easily be used to regenerate $\varphi_{i}$.

Step 4-Elitism: According to the costs evaluated by $\max \left|\mathbf{x}^{\prime}(\boldsymbol{\Phi})\right|$, this study places the $T$ chromosomes with the lowest costs into the mating pool. This ensures that each generation retains better chromosomes.

Step 5-Repeat/End: Repeat steps 1-4 until the number of generations is $G$. Finally, the chromosome with the lowest cost is selected to be the rotation phase factor in the PTS scheme.

\subsection{Modified RVGA PTS}

For practical implementation, rotation phases that can be chosen should set a finite number of phases. The modified RVGA (MRVGA) inserts an additional step between steps 3 and 4 of the RVGA intending to map each continuous phase $\varphi_{i}$ into a set of finite numbers of allowable rotation phases. Taking a set of $W$ allowable phases as an example, i.e., $\phi_{i}^{\prime} \in\{2 k \pi / W \mid k=0,1, \ldots, W-1\}$, continuous rotation phases $\varphi_{i}$ can be mapped to allowable rotation phases $\phi_{i}^{\prime}$ 
based on the mapping function expressed as

$$
\phi_{i}^{\prime}= \begin{cases}2 k \pi / W, & \text { if }(2 k-1) \pi / W \leq \phi_{i}<(2 k+1) \pi / W_{(8)} \\ 0, & \text { otherwise }\end{cases}
$$

\subsection{Modified RVGA PTS with extinction and immigration}

Conventionally, the GA suffers from close breeding. As the number of chromosomes in the mating pool associated with smaller costs grows exponentially, after some generations, the $T$ parent chromosomes chosen to mate are eventually almost identical. If two parents are identical, their children will also be identical and no new information will be disseminated. This study adopts the strategy of Extinction and Immigration (EI) to react against the aforementioned problems [15]. By operations of extinction and immigration, the strategy of EI functions like a particular time varying mutation probability in which $p_{m}$ is close to 1 at the beginning of each new era and then gets smaller for the remaining generations.

Extinction eliminates all of the chromosomes in the current generation except for the chromosome corresponding to the minimum cost. Immigration randomly generates $(P$ -1) chromosomes to propagate the population (a mass immigration). ( $T$ - 1) chromosomes associated with the least costs among these immigrants are then selected as the parents. Together with the surviving chromosome, these are allowed to mate as usual to form the next generation. Generally, there are two cases when extinction and immigration will occur. One is the case when all of the $T$ parents are the same, and the other is the case when no further decrease in the cost values has been reached. This study adopts the second case to determine when to execute the strategy of extinction and immigration.

\section{Numerical results}

This section presents the simulation results of a variety of suboptimal PTS algorithms. In the conducted computer simulations, $10^{5}$ independent OFDM symbols were randomly generated, and all subcarriers with QPSK modulation were divided into eight subblocks with adjacent partition [3]. The simulation parameters are summarized in Table 1. When the EI strategy is not executed in the RVGA method, the size of the mating pool $(T)$ is set at 10 while it is set at 4 when the EI strategy is executed. The optimal combination of the rotation phase vector is to exhaustively locate the minimum PAPR, which requires a full enumeration of the cost function for all possible combinations of phase vectors. The suboptimal methods only execute a partial enumeration of cost function for a subset of all possible combinations of phase vectors.

Figure 2 shows the variation in PAPR complementary cumulative distribution function $(\mathrm{CCDF})$, defined as $F(\xi)$
Table 1 Summaries of simulation parameters

\begin{tabular}{ll}
\hline Parameters & Value \\
\hline Subcarriers number $(K)$ & 64,128 \\
Subblock number $(M)$ & 8 \\
Number of phases $(M)$ & 4 \\
Oversampling factor $\left(f_{s}\right)$ & 4 \\
Population size $(P)$ & 200 \\
Generations $(G)$ & 20 \\
Crossover probability $\left(p_{c}\right)$ & 0.6 \\
Mutation probability $\left(p_{m}\right)$ & 0.1 \\
\hline
\end{tabular}

$=\operatorname{Pr}\left[\operatorname{PAPR}\left(\mathbf{x}^{\prime}(\boldsymbol{\Phi})\right)>\xi\right]$, of the RVGA and the MRVGA methods with different numbers of generations for OFDM systems with 64 subcarriers. Figure 2 shows that the PAPR reduction tends to increase as the number of generations increases. With the requirement of PAPR CCDF equal to $10^{-3}$, the RVGA PTS obtains 6.08 and $5.62 \mathrm{~dB}$ PAPR with reduced computational loads of $6.1 \%(1,000 / 16,384)$ and $24.4 \%(4,000 / 16,384)$, of the computational load required by the exhaustive PTS, respectively. The RVGA searches the rotation phase vector to reduce the PAPR in a continuous domain, and therefore, its PAPR statistic is superior to that of the exhaustive PTS scheme. However, the excellent PAPR reduction performance achieved by the RVGA PTS is not practical because the transmitter must spend large side information to notify the receiver about the rotation phase vector taken at the transmitter. Conversely, the MRVGA PTS is practical, but it suffers from a performance degradation that mainly comes from close breeding in GA and the quantization error in (8).

To compensate for the problems in the MRVGA, the strategy of EI is executed in the MRVGA when no further decrease in cost values has been reached. Figure 3 shows the variation in PAPR CCDF of the MRVGA PTS and the MRVGA PTS with EI strategy (MRVGA_EI) with different numbers of generations for OFDM systems with 64 subcarriers. Figure 3 shows that the PAPR CCDF of the MRVGA_EI PTS with $G=20$ nearly approaches that of the optimal exhaustive PTS. With a similar computational load, the PAPR statistic of the MRVGA_EI PTS with $G=20$ is compared with that of other suboptimal PTS methods.

Figure 4 shows the PAPR CCDFs of various suboptimal PTS schemes, including the proposed MRVGA_EI, the exhaustive search, the iterative flipping (IF) [4], the gradient descent (GD) [5], the simulated annealing (SA) [6], the BGA [7], and the artificial bee colony (ABC) [11], for $N=64$ subcarriers, in which the GD is with parameters $r=3$ and $I=2$ and both the SA and the BGA are with the same parameters in [6] and [7], respectively. Furthermore, the number of enumerations is 4,000 in the SA while the population is 200 and the 


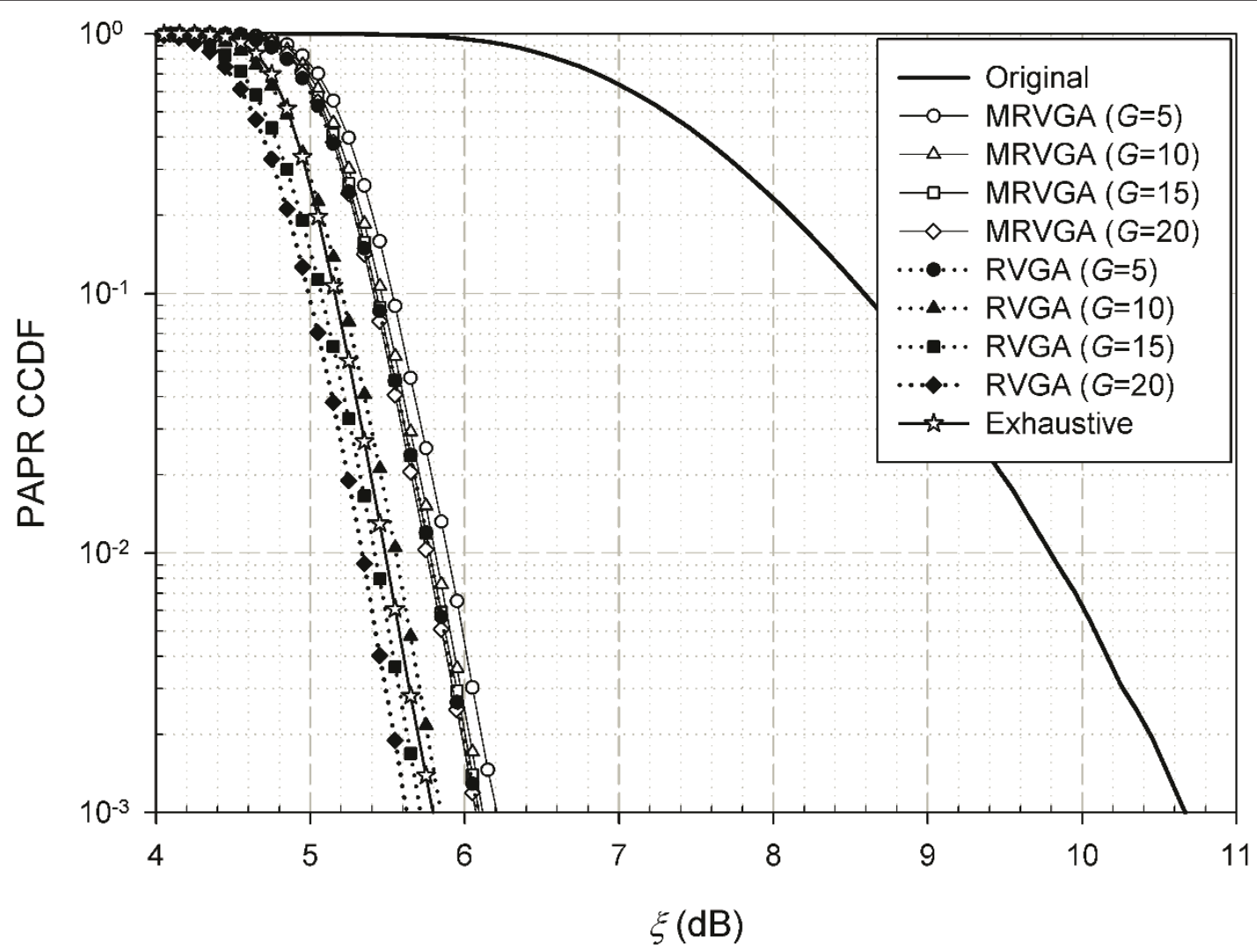

Figure 2 Comparison of the PAPR CCDF of RVGA and MRVGA for different numbers of generations for OFDM systems with 64 subcarriers.

number of the generations is 20 in both of the BGA and the $\mathrm{ABC}$ to ensure having a similar computational load. Figure 4 shows that the value $\xi$ of the original OFDM signal, the IF, the BGA, the SA, the GD, the ABC, the proposed MRVGA_EI, and the exhaustive PTS when the PAPR CCDF equals $10^{-3}$ are 10.66, 7.66, 6.11, 6.02, 5.98, $5.90,5.85$, and $5.8 \mathrm{~dB}$. The results described above show that the proposed MRVGA_EI method performs with almost the same PAPR reduction as that of the exhaustive PTS. However, only approximately $6.1 \%$ computational load is required for the proposed MRVGA_EI PTS method than for the exhaustive PTS.

Figure 5 shows the PAPR CCDFs of considered suboptimal PTS schemes for $N=128$ subcarriers. Figure 5 shows that the value $\xi$ of the original OFDM signal, the IF, the BGA, the SA, the GD, the ABC, the proposed MRVGA.EI, and the exhaustive PTS when the PAPR CCDF equals $10^{-3}$ are $11.06,8.15,6.74,6.68$, $6.6,6.56,6.48$, and $6.41 \mathrm{~dB}$. The results described above again show that the proposed MRVGA_EI method provides nearly with the same PAPR statistic as that of the exhaustive PTS with a lower computational load.
With a similar computational load, the PAPR reduction performance, represented as $\xi$ when $\operatorname{Pr}[\mathrm{PAPR}$ $\left.\left(\mathbf{x}^{\prime}(\boldsymbol{\Phi})\right)>\xi\right]=10^{-3}$, of those considered suboptimal PTS methods are summarized in Table 2. The IF PTS lowers the complexity, but severely degrades PAPR reduction performance. Conversely, the exhaustive PTS yields optimal performance with the highest complexity. The GD, SA, and the BGA PTSs performed more effectively than the IF method, but their complexity is higher than the IF PTS. However, the GD, the SA, and the BGA PTSs are less complex than the exhaustive PTS with more favorable performance than the IF PTS. The proposed MRVGA_EI PTS performs more effectively than the GD, the SA, and the BGA PTSs with the same complexity.

Finally, comparisons of the PAPR reduction performance and complexity trade-offs for the MRVGA_EI, the BGA, the SA, the GD, and the ABC PTS methods are provided in Figure 6, where the value $\xi$ is plotted as a function of the number of enumerations required to achieve $\operatorname{Pr}\left\{\operatorname{PAPR}\left(\mathbf{x}^{\prime}(\boldsymbol{\Phi})\right)>\xi\right\}=10^{-3}$. The GD method shows a limitation in decreasing PAPR with the increase of the number of enumerations for both cases of $r=2$ 


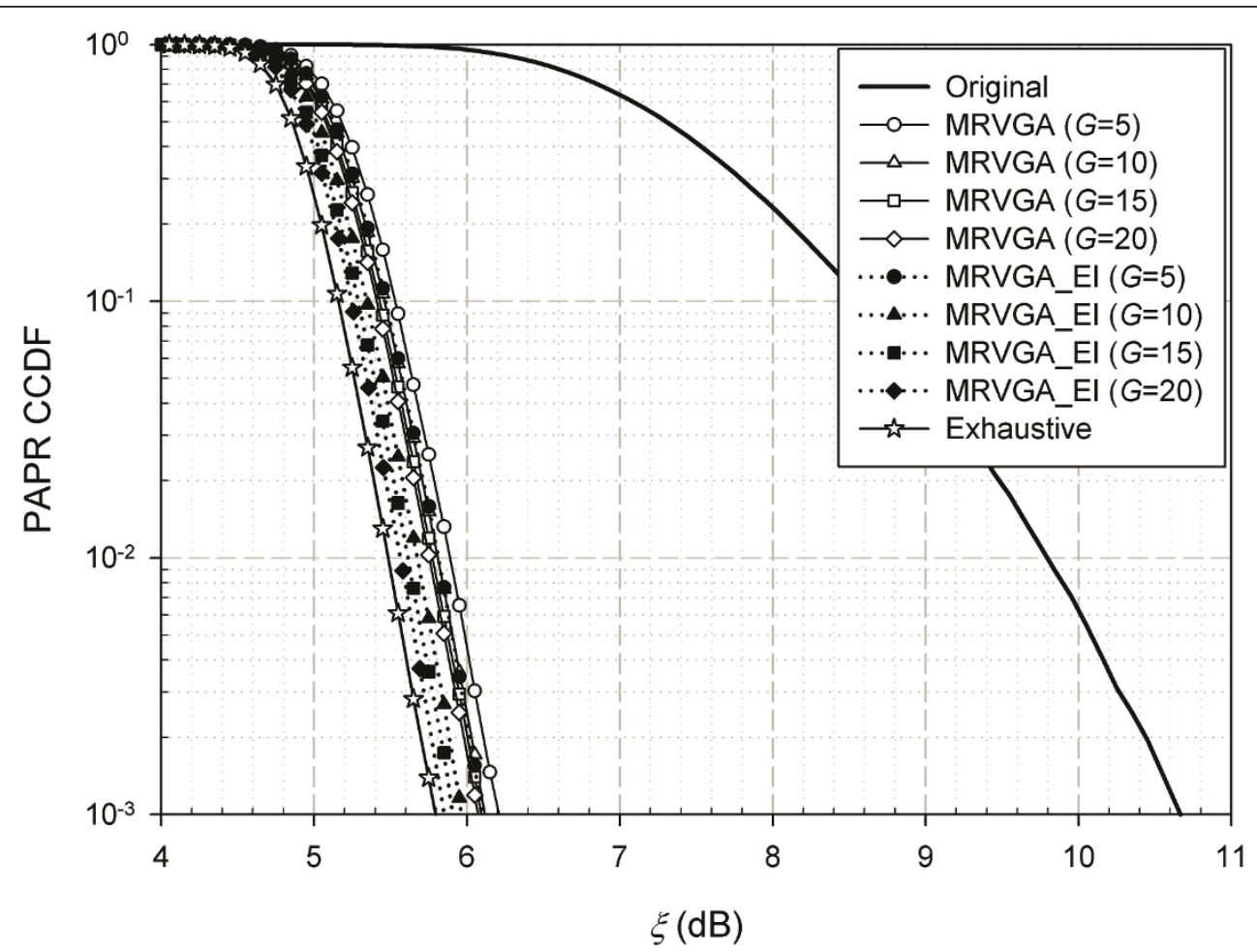

Figure 3 Comparison of the PAPR CCDF of MRVGA and MRVGA.El for different numbers of generations for OFDM systems with 64 subcarriers.

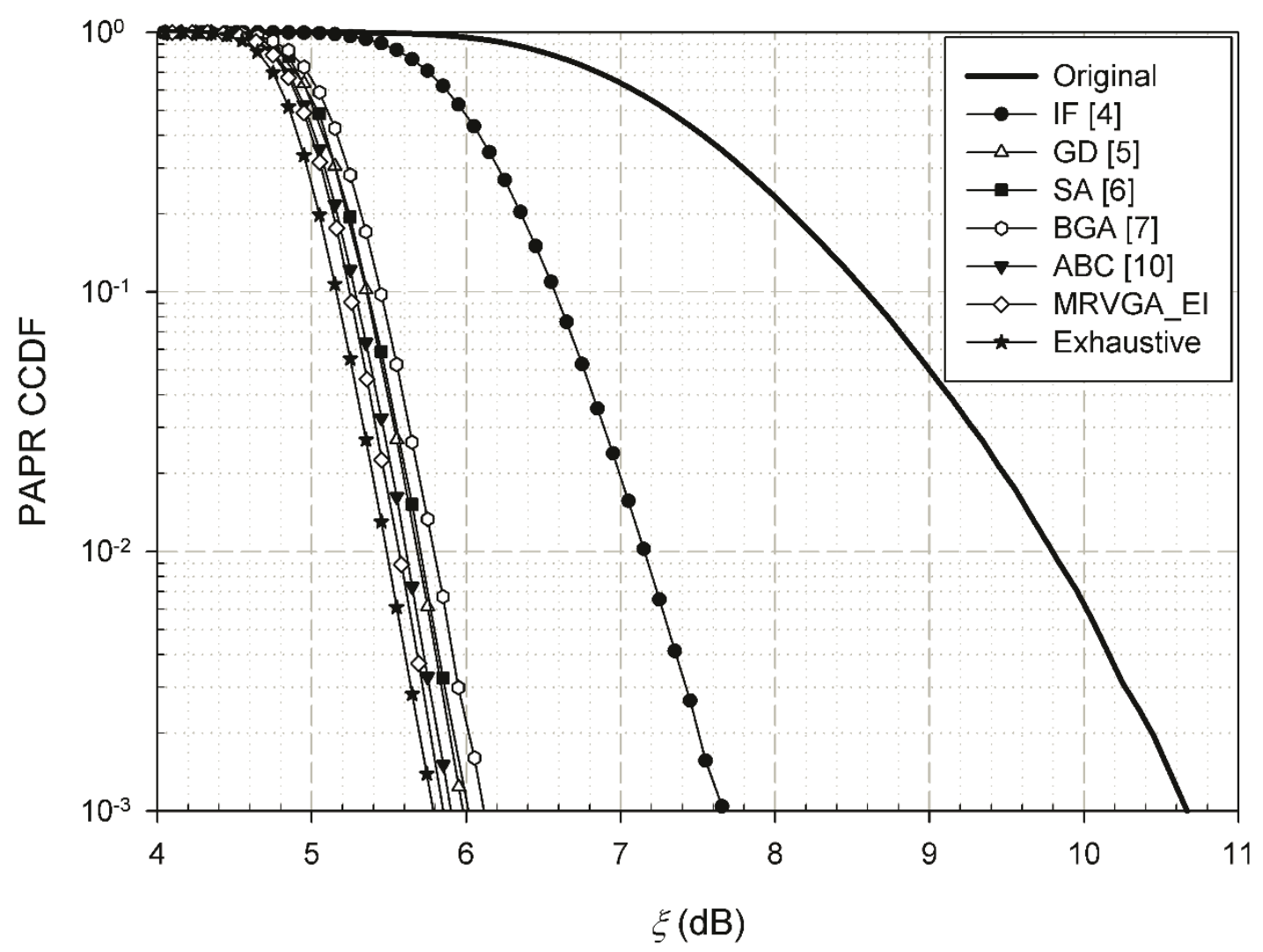

Figure 4 Comparison of the PAPR CCDF of several PTS techniques for OFDM systems with 64 subcarriers. 


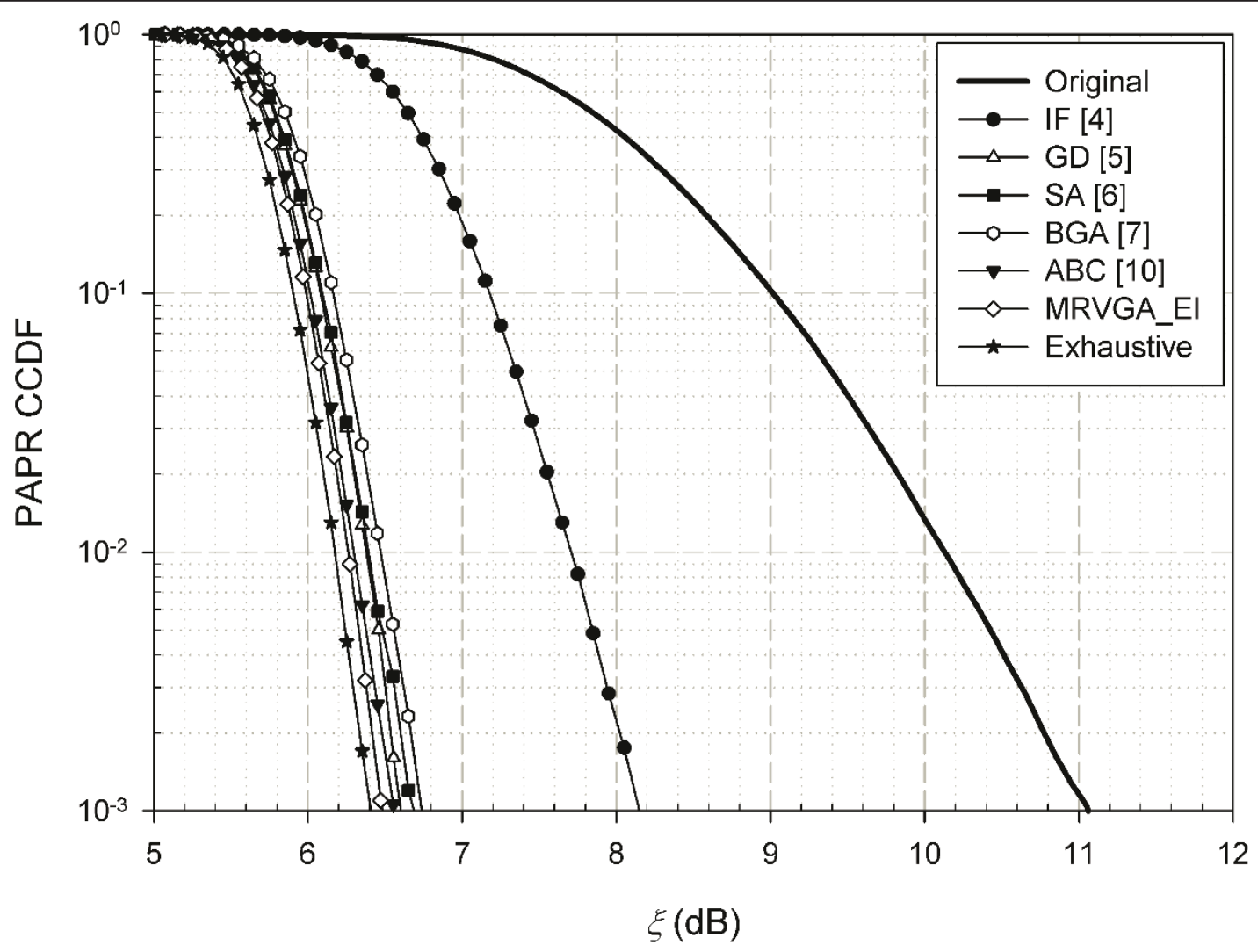

Figure 5 Comparison of the PAPR CCDF of several PTS techniques for OFDM systems with 128 subcarriers.

and $r=3$. With the increase of the number of enumerations, the SA method can converge on a more favorable PAPR reduction performance than that of the BGA method while it exhibits a poorer PAPR reduction performance than that of the BGA method within the region of a low number of enumerations. The ABC outperforms the BGA and the proposed MRVGA_EI within the region of a low number of enumerations, and moreover, it finally converges to a better PAPR reduction than the SA. When the number of enumerations is large enough, the proposed MRVGA_EI PTS not only shows a lower computational load to achieve a specific required PAPR reduction, but also demonstrates its capability of approximately converging to the global optimal solution than other suboptimal methods.

\section{Conclusion}

This paper presents an RVGA method that was used to obtain the rotation phase vector for the PTS technique to reduce the PAPR of OFDM signals. Simulations were conducted and show that the performance of the proposed MRVGA_EI PTS provided almost the same PAPR statistics as that of the optimal exhaustive PTS, while maintaining a low computational load. With the trend that GA hardware is becoming more popular and lowpriced, the proposed MRVGA_EI PTS provides a

Table 2 Comparison between rotation phase-searching schemes

\begin{tabular}{llcc}
\hline Methods & The number of enumerations & $\boldsymbol{\xi}(\boldsymbol{N}=\mathbf{6 4})$ & $\xi(\boldsymbol{N}=\mathbf{1 2 8})$ \\
\hline Exhaustive & $16,384, W^{M-1}$ & 5.80 & 6.41 \\
IF & $28,(M-1) \times W$ & 7.66 & 8.15 \\
GD & $4,480, C_{r}^{M-1} W^{\prime \prime}$ & 5.98 & 6.60 \\
SA & 4,000 & 6.02 & 6.68 \\
ABC & 4,000 & 5.90 & 6.56 \\
BGA & $4,000, P \times G$ & 6.11 & 6.74 \\
MRVGAEEl & $4,000, P \times G$ & 5.85 & 6.48 \\
\hline
\end{tabular}




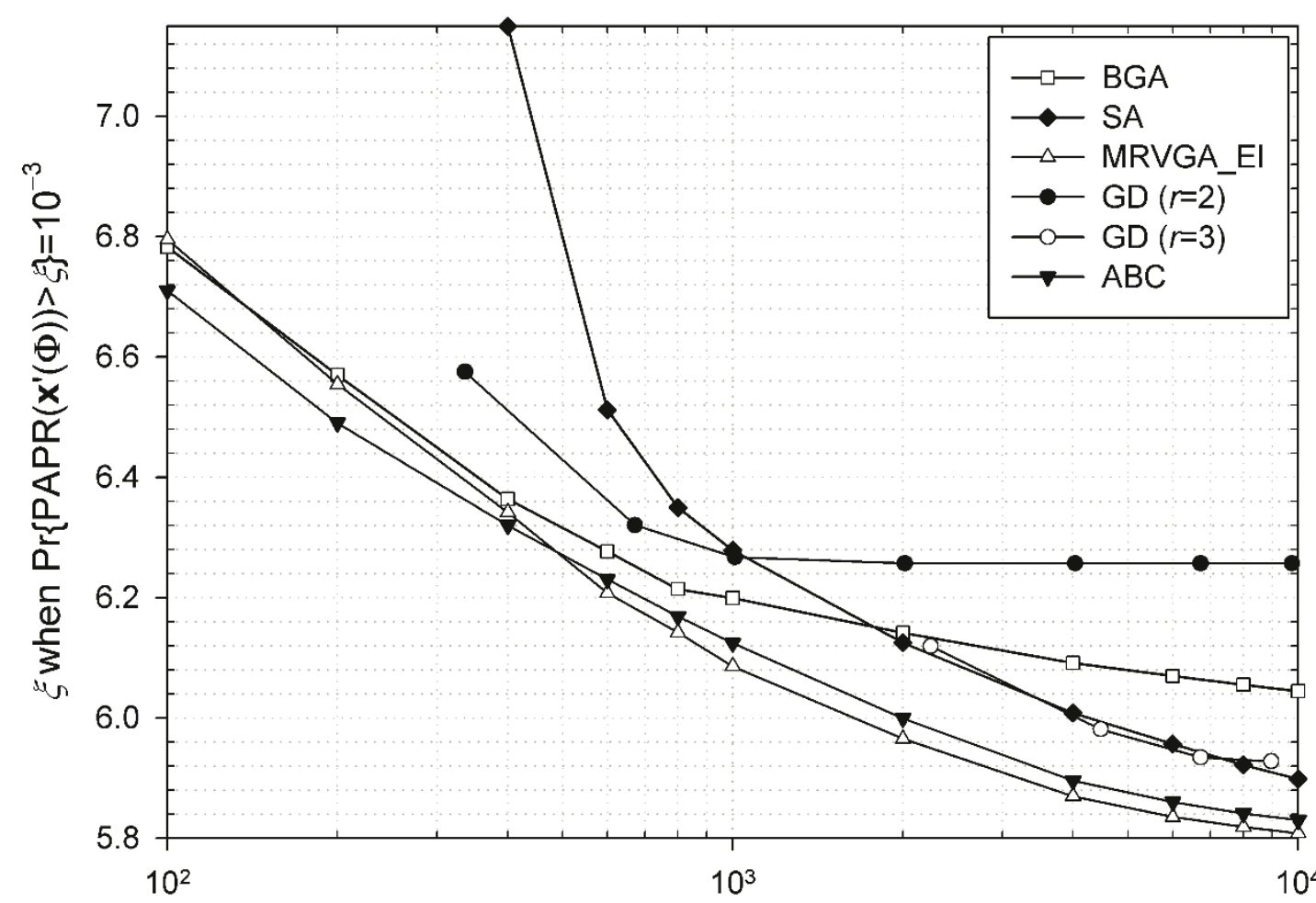

Number of enumeration

Figure $6 \xi$ when $\operatorname{Pr}\left\{\operatorname{PAPR}\left(x^{\prime}(\Phi)\right)>\xi\right\}=10^{-3}$ versus the number of enumerations for OFDM systems with 64 subcarriers.

practical and economical approach toward solving the difficulty of high PAPR in OFDM systems.

\section{Acknowledgments}

This work was supported by National Science Council of Taiwan under Contract NSC98-2221-E-224-019-MY3.

\section{Competing interests}

The authors declare that they have no competing interests.

Received: 16 May 2011 Accepted: 11 October 2011

Published: 11 October 2011

\section{References}

1. R Chang, Synthesis of band-limited orthogonal signals for multichannel data transmission. Bell Syst Tech J. 45(10), 1775-1796 (1996)

2. S Han, J Lee, An overview of peak-to-average power ratio reduction techniques for multicarrier transmission. IEEE Trans Wirel Commun. 12(2), 56-65 (2005). doi:10.1109/MWC.2005.1421929

3. S Muller, J Huber, OFDM with reduced peak-to-average power ratio by optimum combination of partial transmit sequences. Electron Lett. 33(5), 368-369 (1997). doi:10.1049/el:19970266

4. LJ Cimini, NR Sollenberger, Peak-to-average power ratio reduction of an OFDM signal using partial transmit sequences. IEEE Commun Lett. 4(3), 86-88 (2000). doi:10.1109/4234.831033

5. $\mathrm{SH}$ Han, JH Lee, PAPR reduction of OFDM signals using a reduced complexity PTS technique. IEEE Trans Signal Process. 11(11), 887-890 (2004). doi:10.1109/LSP.2004.833490

6. T Jiang, W Xiang, P Richardson, J Guo, G Zhu, PAPR reduction of OFDM signals using partial transmit sequences with low computational complexity. IEEE Trans Broadcast. 53(3), 719-724 (2007)
7. S Kim, M Kim, T Gulliver, PAPR reduction of OFDM signals using genetic algorithm PTS technique. IEICE Trans Commun. E91-B(4), 1194-1197 (2008). doi:10.1093/ietcom/e91-b.4.1194

8. H Liang, Y Chen, Y Huang, C Cheng, in A Modified Genetic Algorithm PTS Technique for PAPR Reduction in OFDM Systems 15th Asia-Pacific Conference on Communications, APCC 2009, 182-185 (2009)

9. Y Zhang, Q Ni, H Chen, Y Song, in An Intelligent Genetic Algorithm for PAPR Reduction in a Multi-Carrier CDMA Wireless System Wireless communications and Mobile Computing Conference, 2008. IWCMC08. International, 1052-1057 (2008)

10. J-H Wen, S-H Lee, Y-F Huang, H-L Hong, A suboptimal PTS algorithm based on particle swarm optimization technique for PAPR reduction in OFDM systems. EURASIP J Wirel Commun Netw. 2008. Article No. 14

11. Y Wang, W Chen, C Tellambura, A PAPR reduction method based on artificial bee colony algorithm for OFDM signals. IEEE Trans Wirel Commun. 9(10), 2994-2999 (2010)

12. PY Chen, RD Chen, YP Chang, LS Shieh, H Malki, Hardware implementation for a genetic algorithm. IEEE Trans Instrum Meas. 57(4), 699-705 (2008)

13. P Fernando, S Katkoori, D Keymeulen, R Zebulum, A Stoica, Customizable FPGA IP core implementation of a general-purpose genetic algorithm engine. IEEE Trans Evolut Comput. 14(1), 133-149 (2010)

14. R Haupt, S Haupt, Practical Genetic Algorithms (Wiley Online Library, 1998)

15. L Yao, W Sethares, Nonlinear parameter estimation via the genetic algorithm. IEEE Trans Signal Process. 42(4), 927-935 (1994). doi:10.1109/ 78.285655

doi:10.1186/1687-1499-2011-126

Cite this article as: Lain et al:: PAPR reduction of OFDM signals using PTS: a real-valued genetic approach. EURASIP Journal on Wireless Communications and Networking 2011 2011:126. 